Musées, Patrimoine et Culture scientifiques et techniques

$181 \mid 2019$

janvier-février 2019

\title{
Questions de regards
}

Élisabeth Caillet

\section{Q OpenEdition \\ Journals}

Édition électronique

URL : http://journals.openedition.org/ocim/2238

DOI : $10.4000 /$ ocim.2238

ISSN : 2108-646X

Éditeur

OCIM

Édition imprimée

Date de publication : 1 janvier 2019

Pagination : 30-37

ISSN : 0994-1908

Référence électronique

Élisabeth Caillet, "Questions de regards », La Lettre de I'OCIM [En ligne], 181 | 2019, mis en ligne le 01 janvier 2020, consulté le 17 mars 2020. URL : http://journals.openedition.org/ocim/2238 ; DOI :

https://doi.org/10.4000/ocim.2238

Ce document a été généré automatiquement le 17 mars 2020.

Tous droits réservés 


\section{Questions de regards}

\section{Élisabeth Caillet}

Lilian Thuram commente La mort de Sardanapale dans la salle sur le pouvoir.

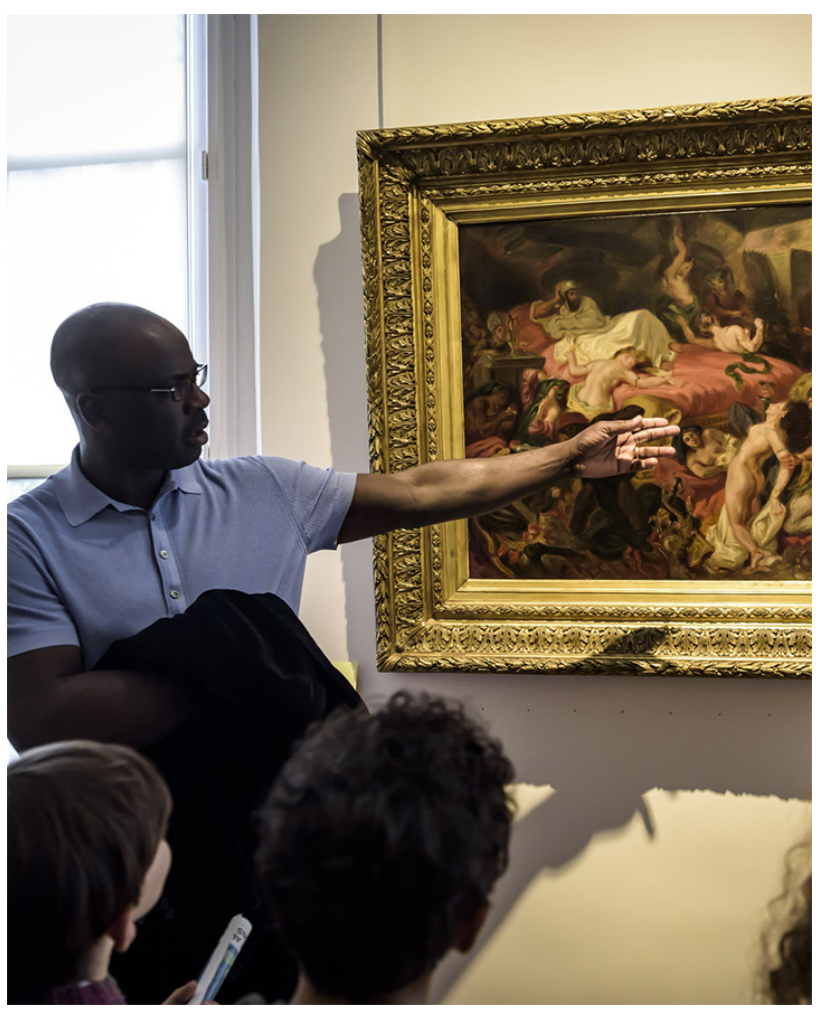

(c) Musée du Louvre/Musée Eugène Delacroix/Olivier Ouadah

1 Les dispositifs de médiation utilisent depuis de nombreuses années le croisement des regards, des démarches artistiques. En effet les œuvres sont commentées non seulement par des paroles ou des textes relevant de l'histoire de l'art ou de l'esthétique, mais encore par d'autres discours artistiques : textes d'écrivains en poésie ou en prose, musique, danse, chanson ${ }^{1}$, autres artistes plasticiens dont l'écriture contemporaine 
tente de faciliter la « présence », la présentation des œuvres exposées, généralement du passé et patrimoniales. Ces différents regards peuvent aussi faire intervenir de nouveaux discours non-experts en histoire de l'art. C'est ce qui été fait avec l'exposition Imaginaires et représentations de l'Orient, Questions de regard(s) qui s'est tenue au musée Delacroix à Paris, du 10 janvier au $1^{\text {er }}$ avril 2018.

\section{Rendre visible ce que l'on ne veut pas voir}

2 La Fondation Lilian Thuram-Éducation contre le racisme a pour objectif de faire " prendre conscience que l'Histoire nous a conditionnés, de génération en génération, à nous voir d'abord comme des Noirs, des Blancs, des Maghrébins, des Asiatiques... Nos différences deviennent des inégalités générées par des mécanismes de domination qu'il est nécessaire de déconstruire ". Ces déconstructions conduisent à questionner les stéréotypes qui structurent nos jugements, à en montrer l'origine historique et à récuser toutes les essentialisations. Les actions de la Fondation prennent de multiples formes : interventions dans les institutions scolaires, outils pédagogiques, livres en particulier pour les enfants, expositions, telle Exhibitions, l'invention du sauvage qui s'est tenue au musée du Quai Branly en 2011 et circule depuis dans le monde entier, Être humain vivre ensemble, exposition itinérante réalisée avec l'association Les Petits débrouillards. La Fondation est une petite structure adossée à un important comité scientifique, dont les membres, très actifs, participent aux actions. Elle existe depuis dix ans.

Lilian Thuram travaille avec des enfants sur l'exposition Nous autres-Vivre ensemble au collège Barbara à Stains.

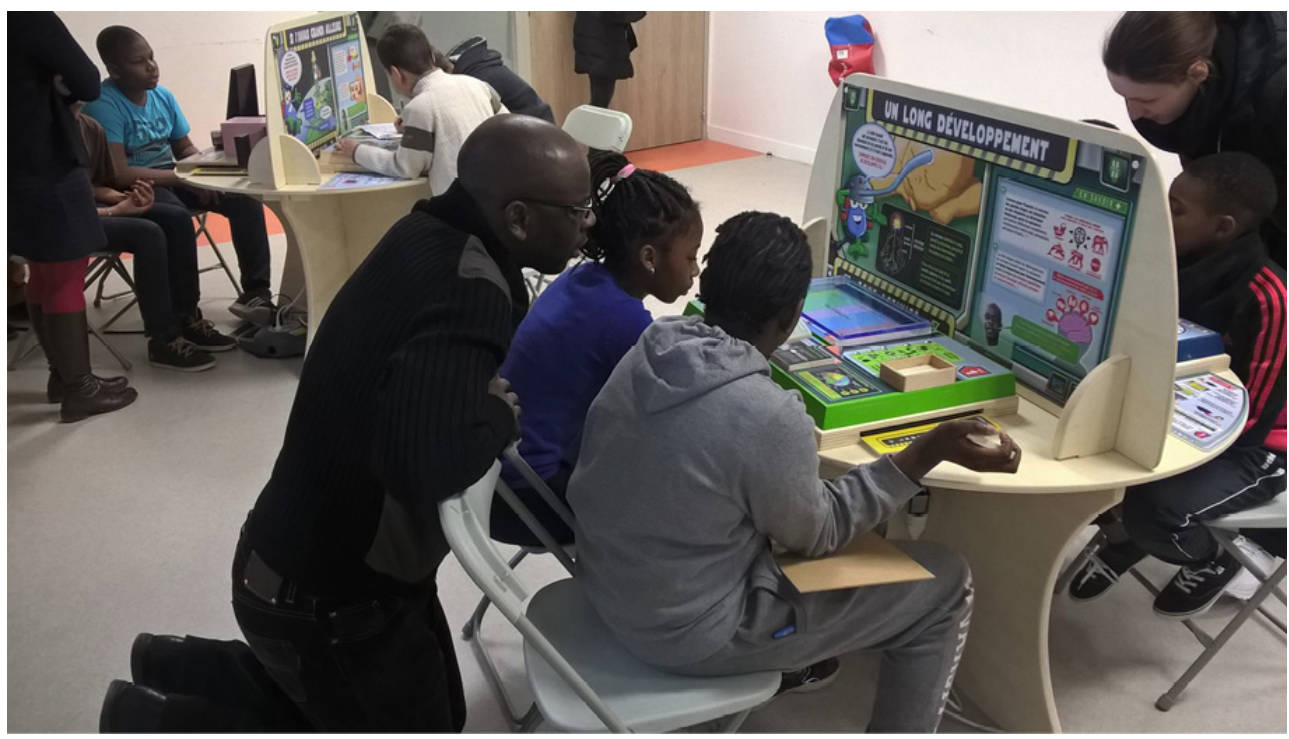

(c) Fondation Lilian Thuram-Éducation/Lionel Gauthier

3 En 2012, Françoise Vergès ${ }^{2}$ politologue et membre du comité scientifique de la Fondation Thuram, choisit une approche originale de certaines œuvres du Louvre : elle proposa de commenter certaines œuvres du musée du Louvre, seule et avec l'historien Marcus Rediker, pour montrer notre aveuglement ordinaire actuel à l'égard des noirs dans la peinture : car nous ne voyons que ce que nous cherchons à voir. Certains éléments des compositions picturales, bien que très précisément disposés par l'artiste, 
sont invisibles à nos yeux mal informés. Nous n'avons pas l'habitude de situer certaines œuvres dans le contexte historique et politique qui fut le leur et dans lequel l'auteur les a lui-même situées : à titre d'exemple, le célèbre tableau de Théodore Géricault, Le Radeau de la Méduse, fut alors regardé à partir de la position du peintre contre la traite des Noirs qui, malgré l'abolition, continuait en particulier au Sénégal où se rendait $L a$ Méduse $^{3}$. De la même manière les natures mortes ou les tableaux de genre qui furent peints entre $1793^{4}$ et $1848^{5}$ font connaître les produits issus de l'esclavage et de la colonisation, produits que nous ne voyons plus ainsi aujourd'hui même si, à leur époque, ils y faisaient explicitement référence : sucre, tabac, café, chocolat, coton, bois précieux... L'intérêt suscité par ces visites effectuées par d'autres médiateurs que ceux issus de l'habituelle histoire de l'art montrait qu'une telle relecture permettait d'attirer d'autres regardeurs, de toucher d'autres publics.

\section{Une adresse au public}

De nombreuses personnes ne possèdent pas les clés de compréhension des œuvres et ne connaissent pas les artistes que présentent les musées de France. Or les professionnels des musées souhaitent que ces œuvres et ces artistes leur permettent de mieux comprendre le monde dans lequel elles vivent. Les approches habituelles sont souvent centrées sur l'histoire de l'art ou sur des contextes esthétiques qui ne les intéressent pas. Les intéresser à ces œuvres et à ces artistes apparaît donc comme essentiel pour leur apprendre à sortir de leurs jugements de valeur, à lutter contre leurs préjugés. Grâce à ce travail, elles peuvent prendre conscience de la façon dont nos préconceptions ont été construites à travers nos histoires et leurs représentations, en particulier picturales. Ces histoires ont donné lieu à des représentations déposées dans les propositions qui sont celles des artistes, dans des œuvres aujourd'hui devenues patrimoniales et sont notre bien commun, capables donc de fonder une relation entre tous les regardeurs. C'est pourquoi il a semblé utile au musée Delacroix, dans ce partenariat avec la Fondation Lilian Thuram, d'apprendre à regarder ces œuvres d'une autre façon. Apprendre à porter un autre regard sur ces œuvres passait par la construction d'un parcours de médiation qui s'attache à mettre en évidence les traces visibles de nos préconceptions et du rôle des artistes pour les diffuser.

Ces nouvelles façons de faire parient sur la possibilité des œuvres d'art à susciter de nouvelles interrogations pour peu qu'on les fasse voir en les replaçant dans leur contexte socio-politique. Les rendre contemporaines de leur époque engage vers un regard qui lui aussi s'interroge sur la construction qu'il produit quand il regarde : si le regardeur est actif, selon un mantra aujourd'hui fortement répété, c'est parce que regarder n'est jamais innocent, mais toujours armé de jugements dont le travail de médiation cherche à susciter la prise de conscience. Un public non averti ne possède sans doute pas les savoirs académiques lui permettant d'entrer facilement dans un travail artistique. Le conduire à s'affranchir de l'obligation de posséder un tel savoir lui permet de constater qu'il possède d'autres clés d'entrée, et ces clés là sont autant de moyens pour qu'il s'aperçoive de ce qu'il sait dans une mise à distance opérante sur son propre regard sur le monde dans lequel il vit. Le plaisir que l'on prend alors à une telle prise de conscience doit apparaître si l'on veut que l'expérience de visite engage à effectuer d'autres visites. Il fut manifeste chez la plupart des visiteurs qui sont venus au 
musée Delacroix. «Je suis comblée! » s'exclame une visiteuse. «Le dispositif s'adresse à des béotiens comme nous ", dit un autre. Pari réussi?

\section{Un accrochage de médiation}

De quoi s'agissait-il donc ? À partir de l'expérience conduite au Louvre autour de l'esclavage Françoise Vergès et moi-même, également membre du comité scientifique de la Fondation Lilian Thuram, eûmes l'idée de monter une action de relecture d'œuvres du musée Delacroix. Nous imaginions alors garder l'accrochage habituel du musée ; mais Dominique de Font-Réault, directrice du musée Delacroix, enthousiaste, nous proposa de réaliser un accrochage-médiation, comme elle venait de le faire avec l'écrivaine Christine Angot.

7 Nous avons alors proposé de travailler sur la question de l'Orientalisme, construction idéologique dont les éléments sont, à notre avis, toujours à l'œuvre aujourd'hui dans la société française. Fidèles à nos objectifs, nous souhaitions mettre en lumière les processus de construction des préjugés à travers lesquels nous regardons les autres, ici en particulier ces " orientaux » qui faisaient tant rêver les romantiques, mais qui aujourd'hui sont jugés comme des menaces pour notre " civilisation ». À partir d'une discussion liminaire avec le personnel du musée, d'une visite des réserves et d'une recherche sur l'Orientalisme ${ }^{6}$ et les idées qui pourraient être mises en avant, nous avons sélectionné une quarantaine d'œuvres de la collection du musée. L'accrochage de ces œuvres a été thématisé autour des idées suivantes : la femme, le pouvoir, le travestissement, le voyage. Les quatre salles du musée réunissaient des œuvres de Delacroix et des objets rapportés lors de son voyage au Maroc en 1832 , des œuvres d'autres artistes qui avaient repris certaines de ses œuvres à leur manière (Frédéric Villot, Hippolyte Portelet, Léon Riesener, artistes cadets de Delacroix...) ainsi que trois types de commentaires : des cartels traditionnels (d'inspiration histoire de l'art) rédigés par le musée, des cartels rédigés par Lilian Thuram et des textes placés sur des bannières précisant la thématique de chacune des salles, textes rédigés par Françoise Vergès. À cette médiation écrite in situ s'ajoutaient un livret de visite qui reprenait les textes rédigés à deux mains par Dominique de Font-Réault d'une part et par Françoise Vergès d'autre part, et surtout une série de médiations orales sous forme de visites ${ }^{7}$ effectuées soit par Lilian Thuram accompagné de Dominique de Font-Réault, conservatrice du musée, soit par Françoise Vergès. Ils ont ainsi conduit presque une visite par semaine chacun durant toute l'exposition, ce qui permit de toucher directement plusieurs centaines de visiteurs.

8 Quelques exemples : la salle sur le travestissement, où l'on pouvait voir des européens se déguiser en orientaux, conduisait les visiteurs à s'interroger : est-ce que les Africains se déguisaient en européens? Se sentir français, n'est-ce pas un déguisement ? Peut-on exister sans déguisement? 
Des enfants dans la salle sur le déguisement échangent avec Lilian Thuram.

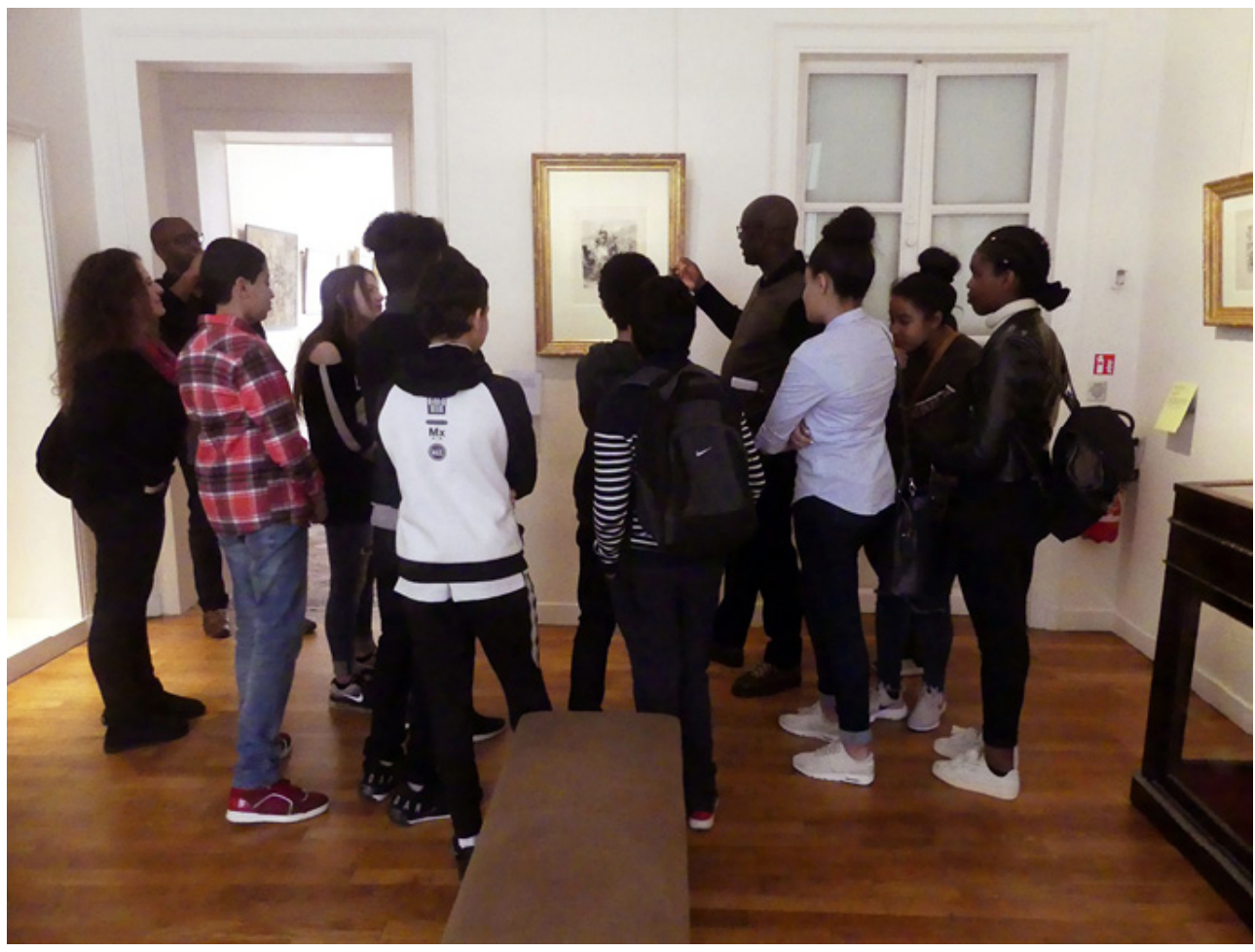

(c) Fondation Lilian Thuram-Éducation/Élisabeth Caillet

9 Autre exemple : pour commenter une gravure de Delacroix intitulée Le combat du Giaour et du Pacha, le musée expliquait que "le sujet de cette gravure est tiré d'un poème de Lord Byron publié en 1813 sous le titre The Giaour A Fragment of Turkish Tale. Il relate les amours malheureux d'un vénitien, le Giaour (surnom péjoratif donné par les Turcs aux nonmusulmans) et d'une esclave, Leila. Delacroix illustre la fin du combat entre le Giaour et le pacha Hassan, maitre de Leila et responsable de sa mort ». De son côté Lilian Thuram écrivait : « rivalité amoureuse d'une part, amour interdit entre un non-musulman et une musulmane. Libre choix du conjoint? Est-on vraiment libre du choix de notre future épouse ou de notre futur époux ? Très souvent non, car on nous conditionne pour que notre conjoint soit de la même religion que nous. Être libre c'est souvent questionner les normes et d'abord les normes familiales. Là est le problème car la famille peut y voir une trahison. Un des aspects de la liberté n'est-il pas de questionner voire de rompre avec les traditions?"».

10 Autre exemple encore : dans la salle sur la femme où était présentée une copie des Femmes d'Alger par Fantin-Latour, Françoise Vergès expliquait qu' " un "Orient des femmes" émerge avec Lady Drummond Hay, Edith Wharton ou Lady Montague dont la description admirative d'un harem en Turquie fera date. Alors qu'en Europe, le corps féminin est corseté, le corps féminin oriental apparaît libre et la danse dite "orientale" offre au corps la possibilité de s'exprimer autrement que dans des gestes étriqués. Le harem fascine et les voyageurs n'ont de cesse de vouloir le "pénétrer". Courtisane voluptueuse, alanguie au milieu d'un riche décor, la femme orientale est objet de désir, un fantasme de femme à la fois fatale et soumise ». 
Des enfants copient Les femmes d'Alger (copie du tableau de Delacroix par Fantin-Latour) dans la salle sur la représentation de la femme.

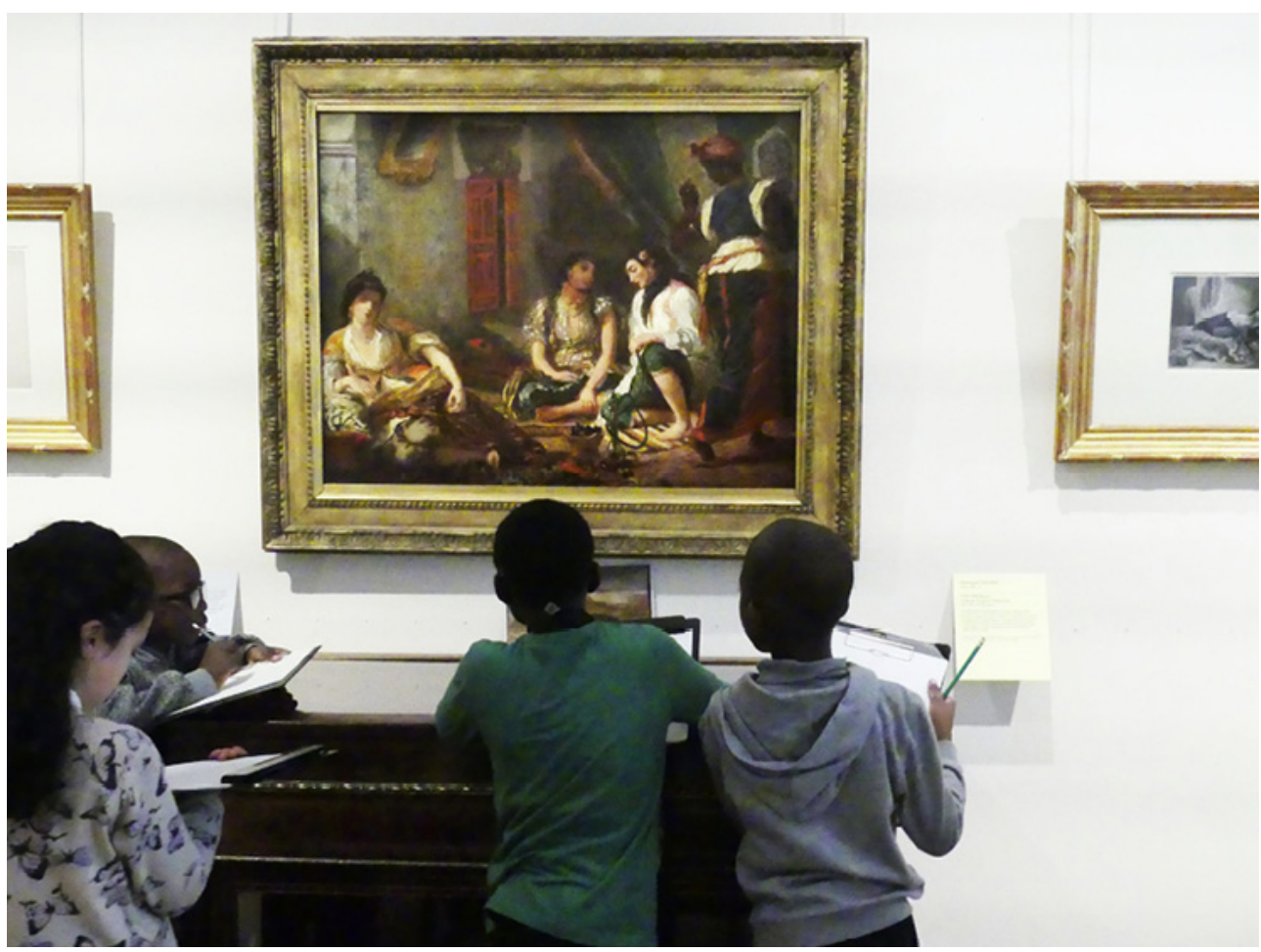

(C) La Rotonde

11 Un dernier exemple montre comment le regard d'un non expert en histoire de l'art permet une actualisation des œuvres : à propos des tableaux représentant des lions (dont on sait par son Journal que Delacroix les a peints à partir de ceux du Muséum national d'Histoire naturelle et pas du tout d'après nature au Maroc, d'où ils avaient disparu), Lilian Thuram faisait remarquer, lors de ses visites, que le surnom de l'équipe nationale de football du Maroc est «Les lions de l'Atlas ». Il orientait alors le regard des visiteurs sur la disparition des espèces et sur la persistance des représentations traditionnelles. "Nous croyons être en 2018 mais nous sommes aussi plongés dans notre passé. Les choses anciennes ne s'effacent pas; elles demeurent dans notre culture partagée. Cela produit souvent des malentendus et parfois des conflits».

\section{Mobiliser tous les médias}

Afin de montrer comment l'Orientalisme avait pénétré tous les arts, un week-end fut organisé à mi-parcours où Gerty Dambury, une comédienne lut des poèmes (Victor Hugo, Byron...), Sarah Aguilar, une chanteuse et Alexandre Peigne, un accordéoniste jouèrent de la musique (Berlioz...).

13 Françoise Vergès mit par ailleurs en place deux visites avec Mehdi Derfoui, un cinéaste ${ }^{8}$ et Kader Attia, un artiste plasticien. La diversité des approches artistiques, croisant autrement ce que nous avions déjà entrelacé dans notre accrochage et dans les événements qui l'accompagnaient, permettait ainsi de mettre en évidence la multiplicité des expressions artistiques ayant contribué à la construction des préjugés sur «l'Orient ». Les visiteurs éprouvaient alors, par les différentes productions 
artistiques que les stéréotypes deviennent des réflexes, sont incorporés et apparaissent alors comme « naturels ».

On pourrait aussi évoquer toutes les autres formes de médiation plus traditionnelles réalisées par les médiateurs et même les personnels de surveillance du musée qui avaient été formés par Lilian Thuram. Le rapport d'évaluation réalisé par l'association Pavages montre bien l'adhésion de ces nouveaux médiateurs au dispositif plus qu'aux propos des concepteurs : "ça permet au public un regard qui complexe moins qu'un conférencier ou qu'un spécialiste et à l'inverse c'est très valorisant pour les agents » dit un agent de surveillance qui réalisait aussi des visites guidées".

L'évaluation fait aussi apparaitre " une grande satisfaction globale (...) qui repose sur une adhésion à la démarche de déconstruction des cuvres et d'une lecture des héritages passés pour éclairer le présent et se déploie dans la complexité d'une expérience de visite qui fait dialoguer les arts et mobilise de nombreux sens $»^{10}$.

Françoise Vergès commente la salle sur la représentation de la femme en odalisque au musée Delacroix.

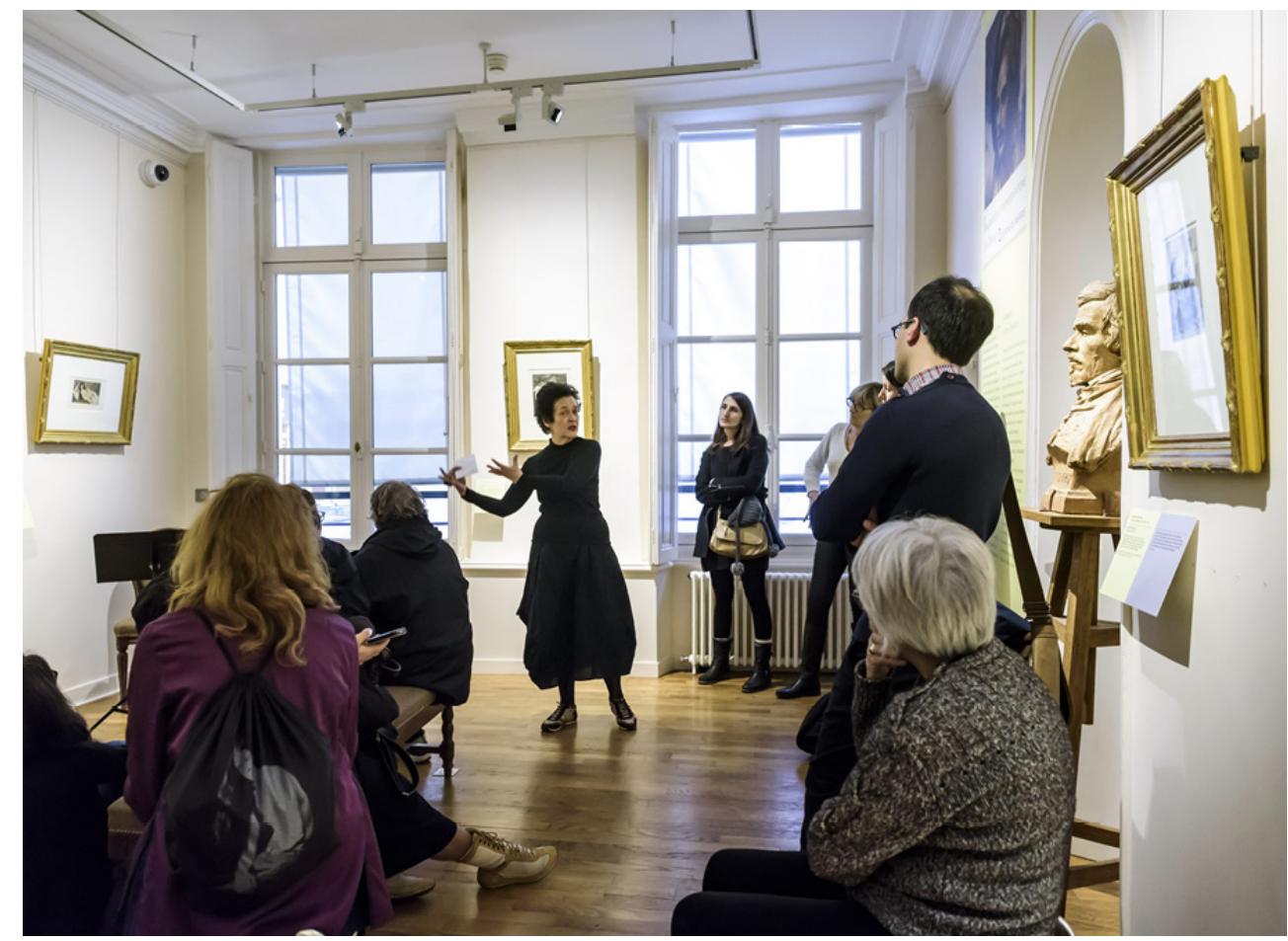

(c) Musée du Louvre/Musée Eugène Delacroix/Olivier Ouadah

\section{Un nouveau public}

L'évaluation qualitative conduite par l'association Pavages a montré qu'une proportion importante de visiteurs était des primo-visiteurs du musée Delacroix, attirés par l'originalité de l'exposition. Plus encore, les modes d'information (twitter, facebook, mails personnalisés auprès des enseignants avec lesquels la Fondation était intervenue lors de projets en classe, communication du musée du Louvre dont dépend le musée Delacroix) sur les visites guidées tant par Lilian Thuram que par Françoise Vergès ont permis de faire venir de nouveaux visiteurs, en particulier des groupes de jeunes 
scolaires. Leur surprise était grande de rencontrer un ancien footballeur prenant le rôle de conférencier d'une visite d'exposition artistique. "C'est sur le nom des personnes impliquées dans la conception de l'événement et sa médiation auprès des publics que sont venus les participants. En miroir de la diversité des commissaires-conférenciers se lit celle des publics drainés. Certains admirent le travail de l'historienne Françoise Vergès, d'autres adhèrent à la reconversion militante de Lilian Thuram et utilisent son statut d'icône populaire pour mobiliser un public du deuxième cercle, tels des élèves ou des proches non familiers des musées $»^{11}$. Avec ces jeunes, en particulier les plus jeunes, les discussions furent nombreuses et animées, les conférenciers se prêtant avec un plaisir non dissimulé à débattre. Rares sont ceux qui contestent la légitimité de Lilian Thuram à s'exprimer sur des œuvres d'art ; bien au contraire ils en apprécient la "simplicité » et la pertinence en termes politiques et historiques. "Il n'y a pas de personne légitime, dit un visiteur, en fait, et c'est ça qui est intéressant. Ça remet en cause le discours du musée, qui est soi-disant légitime et on voit bien que non, il n'y a pas que ça ".

Une autre forme de médiation : les musiciens (Sarah Aguilar et Alexandre Peigne) jouent dans l'atelier de Delacroix qui traite le thème du voyage.

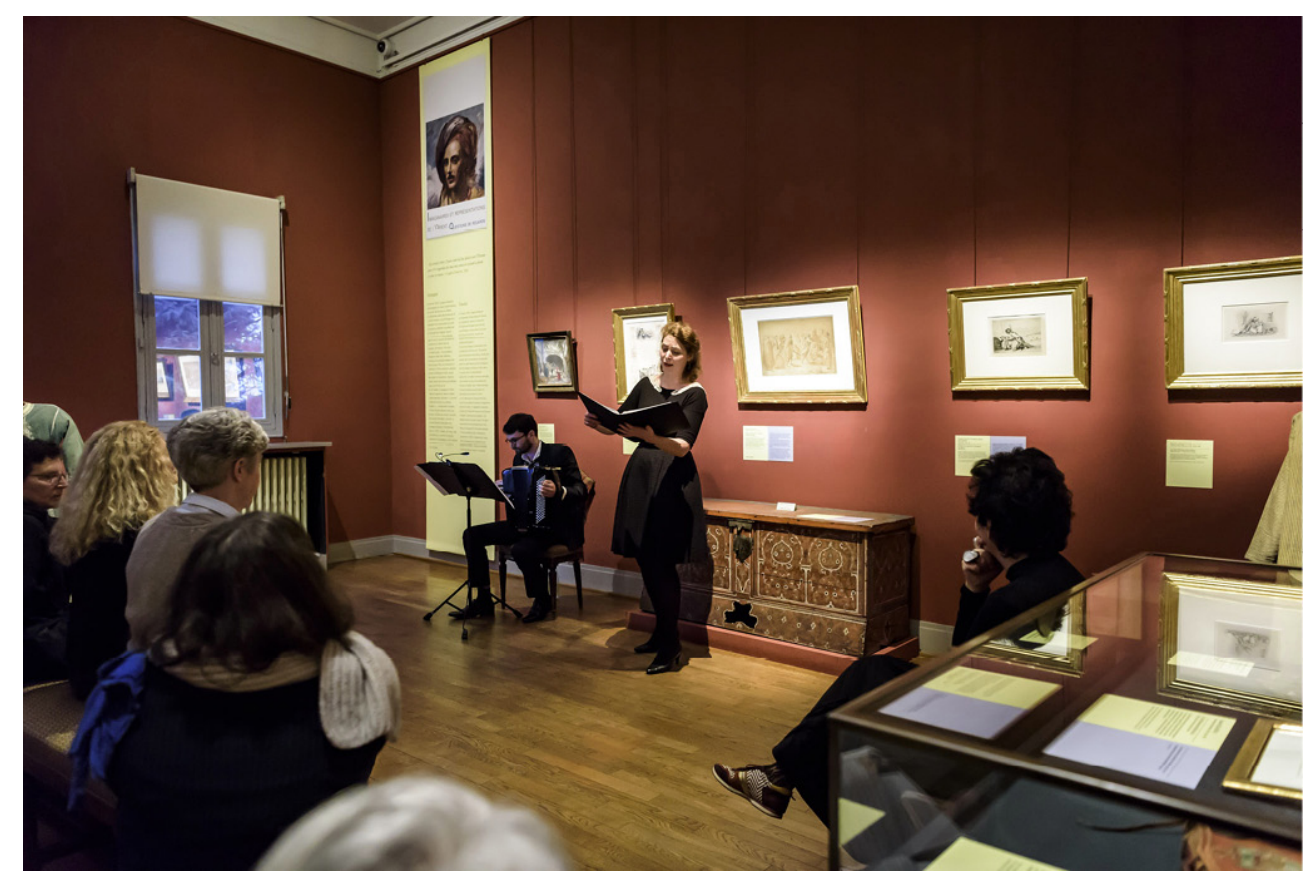

(C) Musée du Louvre/Musée Eugène Delacroix/Olivier Ouadah

\section{II n'y a pas que les blockbusters}

L'évolution des musées montre qu'ils risquent de devoir présenter davantage d'expositions spectaculaires, s'adressant à un public si possible de masse et prêt à payer pour un loisir présenté comme d'exception. Le passage que nous avons observé entre le musée d'expositions permanentes et celui des expositions temporaires se renouvelle aujourd'hui : il semble que la contrainte du spectaculaire va favoriser la tenue de petites expositions légères, qui prendront place entre les grandes expositions blockbusters. Et puis n'oublions pas que beaucoup de petits musées n'ont pas les moyens d'attirer des financements pour de grosses manifestations. Afin d'éviter le repli sur des 
formules telles les expositions dossiers, très prisées des spécialistes experts, il nous semble qu'il soit possible de garder des accrochages pérennes et de ne faire varier que les discours que l'on tient sur les œuvres. Telle pourrait être une nouvelle approche du musée, fondée sur la facilité d'y faire pénétrer des discours, des démarches sensibles ou intellectuelles grâce à des personnes qui auraient à cœur d'y porter un regard adjacent, capable d'intéresser, au sens strict du terme, des regardeurs non férus des savoirs académiques mais forts de savoirs différents.

C'est pourquoi le souhait de la Fondation Thuram est de pouvoir reproduire ailleurs ce type d'expérience, autour de ces collections de musées qui en disent assurément beaucoup plus qu'elles ne veulent le croire.

\section{BIBLIOGRAPHIE}

Frémaux, J. La question d'Orient. Fayard, 2014, 624 p.

Roustan, M. Évaluation qualitative du projet Imaginaires et représentations de l'Orient. Questions de regard(s) au musée national Eugène Delacroix, rapport de synthèse réalisé pour la Direction des Publics de la Direction des Patrimoines du ministère de la Culture, le musée national Eugène Delacroix et la Fondation Lilian Thuram-Éducation contre le racisme, Paris 2018, (non publié), Pavages, $29 \mathrm{p}$.

Serain, F., Vaysse, F., Chazottes, P. et Caillet, É. (dir.) La médiation culturelle : cinquième roue du carrosse? Paris : L'Harmattan, coll. Patrimoines et sociétés, 2016, 270 p.

Vergès, F. L'Homme prédateur, ce que nous enseigne l'esclavage sur notre temps. Paris : Albin Michel, coll. « Bibliothèque Idées ", 2011, 226 p.

Site de la Fondation Lilian Thuram : www.thuram.org/

\section{NOTES}

1. Voir le tout récent clip de Beyoncé et de Jay-Z tourné au Louvre.

2. Chair Global South(s), Collège d'études mondiales, FMSH, Paris.

3. www.canal-u.tv/video/, site consulté le 20 juillet 2018.

4. Date de la première abolition de l'esclavage dans les colonies françaises, à Saint-Domingue.

5. Date de la seconde et définitive abolition de l'esclavage dans les colonies françaises.

6. En particulier à partir du livre de Jacques Frémaux, La question d'Orient. Fayard, 2014.

7. Deux visites par semaine sans compter des moments de présence pour une simple rencontre avec le public.

8. Cf. : www.youtube.com/watch?v=TJ7nwbbIpkc et www.lescahiersdelislam.fr/Rencontre-avecOubayda-Mahfoud-L-image-de-l-Islam-dans-le-cinema_a1245.html; ainsi que le documentaire Hollywood et les Arabes de Jack Shaheen.

9. Rapport d'évaluation de Pavages, sous la direction de Mélanie Roustan, p. 10.

10. Rapport d'évaluation de Pavages, p. 12.

11. Rapport d'évaluation de Pavages, p. 8. 


\section{RÉSUMÉS}

Initié par la Fondation Lilian Thuram-Éducation contre le racisme en partenariat avec le musée Delacroix, le projet présenté ici montre comment, en s'affranchissant des codes habituels de l'histoire de l'art et de l'esthétique, il est possible d'apprendre à regarder des œuvres d'une autre façon et sans préjugés grâce à de nouvelles clés d'entrée et en mobilisant de nouvelles formes de médiation.

INDEX

Mots-clés : Scolaire, médiation

\section{AUTEUR}

\section{ÉLISABETH CAILLET}

Muséologue, membre du comité scientifique de la Fondation Thuram-Éducation contre le racisme elisa.caillet@gmail.com 